\section{P78 PHYSICAL INACTIVITY IS ASSOCIATED WITH MID-THIGH INTRAMUSCULAR FAT IN PATIENTS WITH COPD}

doi:10.1136/thoraxjnl-2012-202678.320

'M Maddocks, ${ }^{2} \mathrm{D}$ Shrikrishna, ' $\mathrm{S}$ Vitoriano, ${ }^{2} \mathrm{RJ}$ Tanner, ${ }^{2} \mathrm{SA}$ Natanek, ${ }^{3} \mathrm{~N}$ Hart, ${ }^{2} \mathrm{PR}$ Kemp, ${ }^{1} \mathrm{~J}$ Moxham, ${ }^{2} \mathrm{Ml}$ Polkey, ${ }^{2} \mathrm{NS}$ Hopkinson. 'King's College London, Departments of Palliative Care, Policy \& Rehabilitation and Asthma, Allergy and Lung Biology, London, UK; ${ }^{2}$ National Heart and Lung Institute, NIHR Respiratory Biomedical Research Unit, Royal Brompton and Harefield NHS Foundation Trust and Imperial College, London, UK; 'uy's and St Thomas' NHS Foundation Trust and King's College London, NIHR Comprehensive Biomedical Research Centre, London, UK

Introduction Quadriceps muscle impairment is an important complication of COPD occurring in mild as well as more advanced disease $^{1}$. This is driven by physical inactivity and can include muscle atrophy and/or a shift towards a less aerobic phenotype with reduced Type I fibre proportions and a reduction in capillarity and oxidative enzymes. ${ }^{2}$ We hypothesised that physical inactivity in COPD patients would be associated with intramuscular fat and that this could potentially be a non-invasive and non-volitional marker of muscle quality.

Methods Mid-thigh cross-sectional area $\left(\mathrm{MT}_{\mathrm{CSA}}\right)$, percentage intramuscular fat and skeletal muscle attenuation (Hounsfield units [HU]) were assessed using computed tomography (CT) image analysis. Tissues were differentiated using standard attenuation ranges; fat: -190 to $-30 \mathrm{HU}$ and skeletal muscle: -29 to $150 \mathrm{HU}$ Quadriceps isometric maximal voluntary contraction (OMVC) was measured using a strain gauge. Fat-free mass index (FFMI) and the impedance ratio $\left(Z_{200} / Z_{5}\right)$ were determined by bioelectrical impedance analysis. Daily step count and physical activity level (PAL) were recorded over 6 days using a multisensory biaxial armband accelerometer (SenseWear, Bodymedia; Pittsburgh, US).

Results 69 patients (mean (SD), 65(8) years, FEV 44(21)\% predicted, 54\% male) participated in the study. Mean (SD) daily step count was 4502 (3274) steps; physical activity level 1.4 (0.2); QMVC 25.2 (5.9) kg; FFMI $17.3(2.3) \mathrm{kg} / \mathrm{m}^{2}, \mathrm{MT}_{\mathrm{CSA}} 178$ (43) $\mathrm{cm}^{2}$. Using a stepwise regression model incorporating $\mathrm{MT}_{\mathrm{CSA}}$, intramuscular fat, skeletal muscle attenuation, QMVC, and FFMI as independent variables, only skeletal muscle attenuation (HU) was retained as an independent correlate of daily step count $(r=0.34, p=0.006)$. In a similar model, percentage intramuscular fat was the only independent predictor of physical activity level $(r=0.37, p=0.002)$. The bioelectrical impedance ratio $\left(Z_{200} / Z_{5}\right)$ was also associated with skeletal muscle attenuation $(r=0.40, p<0.001)$ in this cohort.

Conclusion These data suggest that muscle "quality" assessed using CT is independently associated with daily physical activity and may therefore have potential as a biomarker in this area.

1. Shrikrishna D et al. Quadriceps wasting and physical inactivity in patients with COPD. Eur Resp J 2012; doi: 10.1183/09031936.00170111

2. Shrikrishna D, Hopkinson NS. Chronic obstructive pulmonary disease: consequences beyond the lung. Clin Med 2012; $12: 71-4$

\section{P79 QUANTIFYING PHYSICAL ACTIVITY FOR 30 DAYS VIA PEDOMETRY IN COPD PATIENTS}

doi:10.1136/thoraxinl-2012-202678.321

AD Alahmari, GC Donaldson, ARC Patel, BS Kowlessar, AJ Mackay, R Singh, JA Wedzicha University College London, London, UK

Introduction Physical activity and exercise capacity are among the important patient-relevant parameters in COPD. COPD patients are less active and less likely to go outside when compared with the general population and this worsens during exacerbation (1). The aim of this study was to determine whether step-counts measured by a pedometer (Yamax Digi-walker SW-200) would be a useful method of quantifying physical activity in stable COPD patients on the community.
Methods Stable COPD outpatients wore a pedometer for 30 days, had a six minute walk distance (6MWD) assessed according to ATS protocols and completed the SGRQ (St. George's Respiratory Questionnaire) and MRC dyspnoea score. The pedometer was worn on the left-hand side during waking hours. Patients recorded daily step counts and time spent outdoors on written daily diary cards.

Results Fifty-five COPD patients had a mean ( \pm SD) age 70.3 (8.7) years and FEV1 $51.0 \%$ predicted ( \pm 14.1$)$; male gender $67 \%$. The mean $( \pm \mathrm{SD}) 6 \mathrm{MWD}$ was $416( \pm 88)$ metres, with an average of $4327( \pm 2944)$ steps/day and spent $3.1( \pm 1.4)$ hours outdoors. Figure 1 shows that patients with greater $6 \mathrm{MWD}$ took more steps per day during normal activity $[\mathrm{r}=0.47 ; \mathrm{p}=0.001]$, Fig. $1 \mathrm{~A}$, and stayed outdoors for longer $[r=0.41 ; p=0.003]$, Fig. 1B. Patients with lower step counts tended to have a poorer health status in terms of SGRO score $[r=-0.31 ; p=0.025]$, Fig. $1 \mathrm{C}$, and a higher level of dyspnoea on the MRC score $[R h o=-0.39 ; p=0.005]$, Fig. 1D.

Conclusions Daily step counts measured by a pedometer averaged over 30 days correlated well with objective tests of physical capacity, time outdoors, health status and dyspnoea. Thus pedometry is a useful method of quantifying daily physical activity in COPD patients.

\section{References}

1. Donaldson GC et al., Exacerbations and Time Spent Outdoors in Chronic Obstructive Pulmonary Disease, Am J Resp Crit Care Med (2005) 171: 446-452
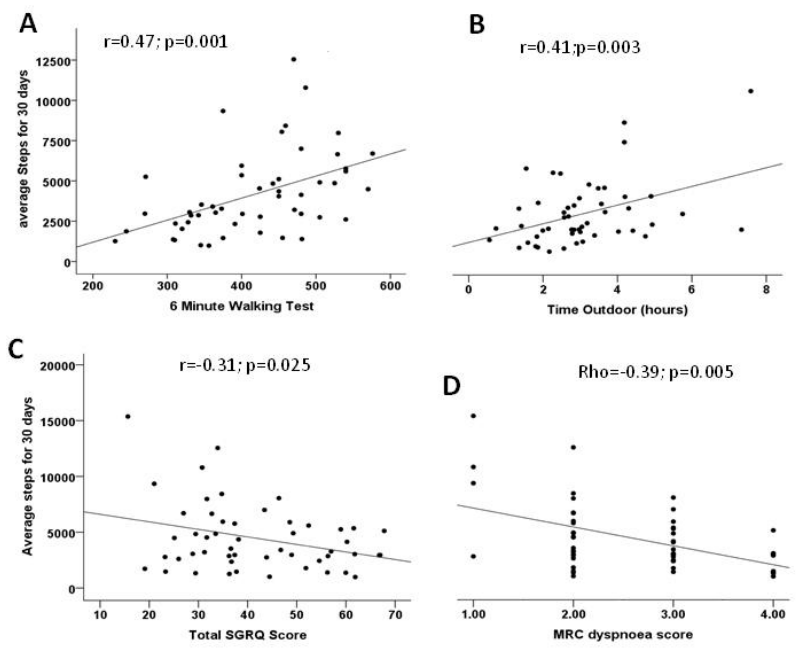

Abstract P79 Figure 1

\section{P80 COMING AND GOING: COPD PATIENTS' EXPERIENCES OF HOSPITAL ADMISSION AND DISCHARGE}

doi:10.1136/thoraxjnl-2012-202678.322

${ }^{1} \mathrm{M}$ Hodson, ${ }^{2} \mathrm{~S}$ Andrews, ${ }^{2} \mathrm{~S}$ Walker, ${ }^{3} \mathrm{M}$ Roberts. ${ }^{1}$ Homerton University Hospital, London, UK; ${ }^{2}$ Department of Acute Care, Anglia Ruskin University, Essex, London; ${ }^{3}$ nstitute of Health Sciences Education, Bartsthe London School of Medicine and Dentistry, London, UK

Background Chronic Obstructive Pulmonary Disease (COPD) is a major cause of emergency hospitalisation in the UK. Identifying the principal decisions of seeking admission to hospital and subsequent experiential aspects for the admission and discharge process make it possible to benchmark future quality service provision for COPD patients.

Aim Part of a larger study about COPD this aspect of the research aimed to identify patients' experiences of being hospitalised with COPD. The focus of this presentation is about patient-reported experiences of making the decision to go to hospital and later, their subsequent discharge from hospital.

Method Nineteen patients with recent acute hospital care for a COPD related illness or exacerbation were interviewed face-to-face. 